\title{
STELLAR EFFECTIVE TEMPERATURES THROUGH THE INFRARED FLUX METHOD
}

\author{
C. MEGESSIER \\ Observatoire de Paris-Meudon \\ F-92195 Meudon Cedex France
}

\begin{abstract}
.
The principle of the method and its advantages are recalled, as well as the works giving such $\mathrm{T}_{\text {eff }}$. Although the method is almost model independent, the monochromatic red fluxes play an important part. The resulting $\mathrm{T}_{\text {eff }}$ depends on the blanketing in the models. The stellar metallicity and gravity have to be known to avoid uncertainties of about $1 \%$. In principle an accuracy around $1 \%$ on $\mathrm{T}_{\text {eff }}$ could be obtained, but the uncertainty on the absolute red flux calibration, due to the unsolved problem of the Vega red flux, prevents this from being achieved.
\end{abstract}

\section{Introduction and principle of the method}

More and more accurate stellar effective temperatures are required, either for atmosphere modelling or to study the internal structure, to better scale the HR diagram, specially in connection with the high accuracy of Hipparcos parallaxes, and so to fix better the evolutionary track for a star. Then we ask: what accuracy is achievable on $\mathrm{T}_{\text {eff }}$, is it possible to obtain effective temperatures within $1 \%$ or better, as asked for and claimed in some works? Why the infrared flux method (IRFM)?

The most direct method to derive $\mathrm{T}_{\text {eff }}$ relies on the Stefan law. It requires the stellar angular diameter $\theta$ and the total flux received from the star, integrated over the whole wavelength range $f$. Indeed :

$$
F=\sigma T^{4} \quad \text { and } \quad \theta=2 R / d \quad \text { so that } \quad f / F=\theta^{2} / 4
$$

where $R$ is the stellar radius and $d$ the stellar distance.

However the angular diameter is known for the brightest stars only and it has a poor accuracy compared to the aim of $\mathrm{T}_{\text {eff }}$ within $1 \%$. As reported 
by Davis (these Proceedings), for a magnitude 3 star the uncertainty is at least $5 \%$, leading to $2.5 \%$ on $\mathrm{T}_{\text {eff }}$.

In their method, Blackwell and Shallis $(1977,1980)$ assume that in the infrared spectrum, the relation (1) holds also for monochromatic wavelengths :

$$
f_{\lambda} / F_{\lambda}=\theta^{2} / 4 \text { (2) } \quad R=F / F_{\lambda}=\sigma T^{4} / F_{\lambda}=f / f_{\lambda}=R_{o b s}
$$

so that the ratio of (1) over (2) leads to (3) where the angular diameter enters no more. The ratio $R_{o b s}$ deduced from the observations is compared to the ratio $\mathrm{R}$ computed from the atmosphere models. Blackwell et al. justify their assumption by several arguments: the small sensitivity of the infra-red flux to $\mathrm{T}_{\text {eff }}$, the smaller blanketing in the infrared, the more certain stellar opacity sources.

First we list the existing works on the IRFM, then we discuss in turn the required observations and models, and the uncertainties they induce on $\mathrm{T}_{\text {eff. }}$.

\section{Stars with effective temperatures through the IRFM}

The groups who derived $T_{\text {eff }}$ through the IRFM are listed in Table 1 for the normal stars, with the type of the stars and their number. Table 2 lists the works on Ap and Am stars.

\section{Influence of the models and of the stellar metallicity and grav- ity}

In the works earlier than 1990 , it was assumed that the ratio $\mathrm{F} / \mathrm{F}_{\lambda}$, where $\mathrm{F}$ is given directly by the Stefan law and $\mathrm{F}_{\lambda}$ by atmosphere models, does not contribute to the uncertainty on $\mathrm{T}_{\text {eff }}$. Meǵessier (1994) showed qualitatively and quantitatively that, in fact, the model itself has an influence on $\mathrm{T}_{\text {eff }}$ derived from a given observed ratio $R_{o b s}$. That is logically explained by the increase of blanketing in the more recent codes. The numerous UV spectral lines absorb flux that is redistributed in the Paschen continuum, which is then enhanced. The near infrared monochromatic fluxes being larger from the more recent codes, the resulting $R=F / F_{\lambda}$ will be smaller. The relation $\mathrm{R}$ vs $\mathrm{T}_{\text {eff }}$ is lowered and then it leads to larger $\mathrm{T}_{\text {eff }}$ for a given $R_{\text {obs }}$. This is illustrated in Table 3, which compares, for few stars, IRFM $\mathrm{T}_{\text {eff }}$ obtained from the same $R_{o b s}$ and from $\mathrm{R}$ ( $\mathrm{T}_{\text {eff }}$ ) for different models. The change in $\mathrm{T}_{\text {eff }}$ is larger than $1 \%$ between the oldest codes considered and ATLAS9, and it can be as large as $350 \mathrm{~K}$, i.e., $2.4 \%$.

Mégessier (1994) showed that the relations $\mathrm{R}\left(\mathrm{T}_{\text {eff }}\right)$ computed for different metallicities and gravities differ, so that using a relation with inappropriate $[M / H]$ or $\log g$ for a star will induce systematic errors in $T_{\text {eff. }}$ This 
TABLE 1. Stars $T_{\text {eff }}$ from the IRFM

\begin{tabular}{lrll}
\hline Authors & N & Type & Class \\
\hline Blackwell and Shallis (1977) & 7 & A0 to F8 & V, III, II, I \\
Blackwell et al. (1980) & 28 & A, F, G & V, IV, III \\
Saxner et al. 1985 & 31 & F, G & V, IV, III \\
Leggett et al. (1986) & 22 & O to M & V, IV, III \\
Mégessier (1988) & 9 & B3 to A2 & V, IV, III \\
Blackwell et al. (1990) & 114 & F to M & V, IV, III \\
Blackwell et al. (1994) & 114 & F to M & V, IV, III \\
Alonso et al. (1994) & 31 & B to M & V, IV, III, II \\
Alonso et al. (1996) & 475 & F to M & V, IV \\
\hline
\end{tabular}

TABLE 2. Stars $T_{\text {eff }}$ from the IRFM for Ap, Am stars

\begin{tabular}{lrlr}
\hline Ap stars & N & Am stars & N \\
\hline Shallis and Blackwell (1979) & 6 & Lane and Lester 1984 & 10 \\
Lanz (1984) & 12 & Mégessier et al. (1990) & 1 \\
Mégessier (1988) & 12 & Mégessier et al. (1993) & 2 \\
& & Smalley (1993) & 25 \\
\hline
\end{tabular}

is illustrated in Table 4 for few stars. The influence of $[\mathrm{M} / \mathrm{H}]$ is larger than that of $\log \mathrm{g}$. It is effective in the same way as the more or less blanketed models. The error on $\mathrm{T}_{\text {eff }}$ is around $1 \%$ and it depends on $\mathrm{T}_{\text {eff }}$.

TABLE 3. IRFM $\mathrm{T}_{\text {eff }}$ from different models

\begin{tabular}{llrrrrrrr}
\hline & & $\mathrm{T}_{a, b}$ & $\mathrm{~T}_{\text {atlas8 }}$ & $\mathrm{T}_{\text {atlas9 }}$ & $\Delta \mathrm{CG}-9$ & $\%$ & $\Delta 9-8$ & $\%$ \\
\hline$\beta$ Sex & B6V & $14505^{a}$ & 14746 & 14860 & 355 & 2.4 & 114 & 0.80 \\
$\alpha$ Leo & B7V & $12025^{a}$ & 12075 & 12145 & 120 & 1.0 & 70 & 0.58 \\
$\gamma$ Lyr & B9III & $9950^{a}$ & 10062 & 10102 & 152 & 1.5 & 40 & 0.40 \\
$\alpha$ CMa & A1V & $9750^{a}$ & 9870 & 9900 & 150 & 1.5 & 30 & 0.30 \\
$\mu$ And & A5V & & 8000 & 7985 & & & -15 & -0.18 \\
$\alpha$ Oph & A5III & & 7890 & 7882 & & & -0.8 & -0.10 \\
45 Boo & F5V & $6663^{b}$ & 6720 & 6765 & 102 & 1.52 & 45 & 0.67 \\
$\pi$ Peg & F5III & $6170^{b}$ & 6220 & 6280 & 110 & 1.77 & 60 & 0.96 \\
\hline
\end{tabular}

${ }^{a} \mathrm{~T}_{\text {eff }}$ from Carbon and Gingerich (1969) models

${ }^{b} \mathrm{~T}_{\text {eff }}$ from old MARC models (Gustafsson et al. 1975) 
TABLE 4. Influence of the model metallicity and gravity on IRFM $\mathrm{T}_{\text {eff }}$ for 8 bright stars

\begin{tabular}{lrrrrrrrrr}
\hline$\longleftarrow$ & \multicolumn{3}{c}{$[\mathrm{M} / \mathrm{H}]$} & & & $\longrightarrow$ & $\longleftarrow$ & $\log \mathrm{g}$ & $\longrightarrow$ \\
\hline $\boldsymbol{\beta}$ & 0.0 & 0.3 & 0.5 & $\Delta_{.0}^{.3}$ & $\Delta_{.0}^{.5}$ & $\%$ & 0.4 & 0.3 & $\Delta_{.4}^{3}$ \\
$\alpha$ Lex & 14860 & 14957 & 15037 & 97 & 177 & 1.18 & 14860 & 14905 & 45 \\
$\gamma$ Lyr & 12145 & 12207 & 12260 & 62 & 115 & 0.94 & 12145 & 12187 & 42 \\
$\alpha$ CMa & 10102 & 10139 & 10172 & 37 & 72 & 0.72 & 10102 & 10080 & -22 \\
$\mu$ And & 7985 & 9940 & 9967 & 40 & 67 & 0.67 & 9900 & 9875 & -25 \\
$\alpha$ Oph & 7882 & 7930 & 7957 & 48 & 75 & 0.95 & 7882 & 7828 & -54 \\
45 Boo & 6845 & 6900 & 6935 & 55 & 90 & 1.30 & 6845 & 6845 & 0 \\
$\pi$ Peg & 6280 & 6336 & 6378 & 56 & 98 & 1.55 & 6280 & 6280 & 0 \\
\hline
\end{tabular}

\section{The required observations and and their accuracy}

The total integrated flux received at the earth $f$ is obtained from the flux distribution, calibrated in the visible at $\lambda 5556 \AA$ by $f_{5556}^{*}$ given in Eq. 4

$$
f_{5556}^{*}=f_{5556}^{V e g a} 10^{-0.4\left(V_{*}-V_{\text {Vega }}\right)}(4) \quad f_{\lambda}=C_{\lambda} 10^{-0.4 m_{\lambda}}
$$

where $f_{5556}^{V e g a}=3.4610^{-11} \mathrm{Wm}^{-2} \mathrm{~nm}^{-1}$ is the reference visual flux calibration (Mégessier 1995) as recalled in my previous talk. The construction of the energy distribution has been presented by Bless (this proceedings). The observed red monochromatic flux is given by Eq. 5 , where $C_{\lambda}$ is the near infrared flux calibration at the wavelength $\lambda$.

The uncertainty on $f$, which is mainly due to the spectrophotometry accuracy, is different in the three wavelength ranges: UV, visible and red. As a whole, it is generally less than $1 \%$ for the brightest stars. (see Mégessier 1997 for a complete discussion of IRFM Teff accuracy). The uncertainty on $f_{5556}^{V e g a}$ is $0.7 \%$ as given in Mégessier (1995). The visual magnitudes $\mathrm{V}$ can be measured to better than $0.01 \mathrm{mag}$. The problem of the accuracy on the red calibration $C_{\lambda}$ has been discussed earlier (Mégessier in this proceedings). If one excludes the problem of the difference between Vega observed flux and model's, $C_{\lambda}$ is known within about $2 \%$ or $3 \%$ and the red magnitudes are measured to 0.02 magnitudes or better. Combining all these uncertainties leads to a relative error on $\mathrm{T}_{\text {eff }}$ less than or equal to $1 \%$. Thus the aim of an accuracy better than $1 \%$ seems to be achieved.

But the values given here are valid for well observed bright stars, specially with good spectrophotometry. Moreover, the error due to the problem of the red calibration has to be added i.e. $2 \%$ or more, depending on the 
red monochromatic wavelength, and if the metallicity and gravity of the star are not certain, one more error has to be taken into account, which can be around $1 \%$ or more.

\section{Conclusion}

The infrared flux method is the closest to the Teff definition, it relies almost not on atmosphere models. Then it should give Teff on an absolute scale. The IRFM Teff accuracy can be as good as $1 \%$, may be somewhat better. Then the method is very attractive.

However several limitations exist. The energy distribution is not available for a large amount of stars, and its measurement is not as easy as that of photometric colours. If it is derived from photometric indices or models, the accuracy is lowered. The method is not totally independent of the models and one should be really confident in the model red fluxes. If the visible flux calibration is well constrained, it is not the case of the red ones and the additional error induced is likely the largest among those mentioned here. Also, the metallicity and the gravity of the stars have to be known.

Then we can retain the advantages of the infrared flux method, but also remember its present limitations. It would provide Teff of stars bright enough, used themselves as references. The problem of the red flux calibration is the most important to solve in oder to get an accuracy around $1 \%$ on Teff.

\section{References}

Alonso A., Arribas S., Martnez-Roger C., 1994, AA 282, 684

Alonso A., Arribas S., Martnez-Roger C., 1996, AAS 117, 227

Blackwell, D.E., Shallis, M.J.,: 1977, MNRAS 180, 177

Blackwell, D.E., Lynas-Gray, A.E., Petford, A.D.:1991, A\&A 245, 567

Blackwell, D.E., Lynas-Gray, A.E., 1994, A\&A 282, 899

Blackwell, D.E., Petford A.D., Shallis, M.J.,1980, A\&A 82, 249

Blackwell, D.E., Petford, A.D., Arribas, S., Haddock, D.J., Selby, M.J.,: 1990, A\&A 232, 410

Bless R.C.,1997, this proceedings

Carbon, D.F., Gingerich, O.: 1969, in: Third Smithsonian Conference on Stellar Atmospheres, MIT press, p.377 (CG69)

Davis, J.,1997, this proceedings

Gustafsson, B., Bell, R.A.,Eriksson, K.E., Nordlund, A. : 1975, A\&A 42, 407

Kurucz, R., 1979, ApJS 40, 1

Kurucz, R.L., 1991a, in Stellar Atmospheres: Beyond Classical Models, eds. L. Crivllari, I. Hubeny, D.G. Hummer, NATO ASI-Series C, vol. 341, p. 441

Kurucz, R.L.,1991, in: Stellar Atmospheres : Beyond Classical Models, NATO ASI Series C, vol. 341, p.441

Lane, M.C. and Lester, J.B., 1984, ApJ 281, 723

Lanz, T., 1984, A\&A 139, 161

Leggett, S.K., Mountain, C.M., Selby, M.J., Blackwell, D.E., Booth, A.J., Haddock, D.J. and Petford, A.D., 1986, A\&A 159, 217 
Mégessier C., 1988, A\&AS 72, 551

Mégessier C., 1994, A\&AS 289, 202

Mégessier C., 1995, A\&AS 296, 771

Mégessier C., 1997, A\&AS (submitted)

Mégessier C, van 't Veer C., 1990, in Evolution of stars: the photospheric abundance connection. IAU Symposium 145 (poster paper edition) Eds G. Michaud and A. Tutukov, p.99

Mégessier C, van 't Veer C., 1993, in Peculiar versus normal phenomena in A-type and related stars. IAU Colloquium 138, ASP Conf. Ser. 44, 208

Saxner M., Hammarbck G., 1985, A\&A, 151, 372

Shallis M.J., Blackwell D.E., 1979, A\&A 79, 48

Smalley, B. 1993, A\&A, 274, 391 